\title{
Projeto de filtros ópticos multicamadas utilizando o PSO
}

\author{
Gilvan Borges, Claudomiro Sales, Marco Sousa e João Costa
}

\begin{abstract}
Resumo-Filtros ópticos são empregados em uma ampla variedade de componentes ópticos, podendo ser aplicados desde sistemas WDM de alta capacidade a sensores ópticos, entre outras aplicações. Este artigo apresenta uma metodologia para projetos de filtros ópticos multicamadas. A técnica de otimização utilizada é o PSO (Particle Swarm Optimization), que tem mostrado ser efetiva na otimização de problemas não lineares e que apresentam descontinuidades. O PSO é empregado neste artigo para encontrar o perfil de índice de refração para que o objetivo de projeto, relacionado à refletividade, possa ser alcançado.
\end{abstract}

Palavras-Chave-Filtros ópticos, filmes finos, multicamadas, particle swarm optimization (PSO).

Abstract-Optic filters are used at a wide variety of optical components, which are applied from high capacity WDM systems to optical sensors, among others applications. This paper presents a methodology for projects of multilayer optic filters. The used optimization technique is the PSO (particle swarm optimization), which has shown to be effective at the optimization of non-linear problems that present discontinuities. In this paper, PSO is used to find the refractive index profile for the project goal, which is related to the reflectivity, can be reached.

Keywords-Optical filter, thin film, multilayer, particle swarm optimization (PSO).

\section{INTRODUÇÃO}

Os filtros ópticos são tipos de estruturas que possuem características especiais de reflexão e transmissão de luz, de tal forma que podem bloquear ou transmitir a luz em uma determinada freqüência, com mais ou menos intensidade. São largamente encontrados em elementos ópticos, dos mais simples como os espelhos, até em equipamentos óptico-eletrônicos complexos, ou ainda em sensores ópticos, com diversas aplicações na indústria, medicina meteorologia, construção civil, dentre outros [1].

Os filtros ópticos baseados em multicamadas, ou filmes finos (termo usado quando as espessuras das camadas são da ordem do comprimento de onda da radiação eletromagnética analisada), são fabricados através da deposição controlada de diferentes materiais dielétricos sobre um substrato [2]. Tipicamente as camadas são constituídas por apenas dois materiais de índices de refração diferentes e alternados. A escolha dos materiais, da quantidade de camadas e da espessura de cada uma delas determina o comportamento do filtro em função da freqüência, do ângulo de incidência e do tipo de polarização da luz.

Gilvan Borges, Claudomiro Sales, Marco Sousa e João Costa, Departamento de Engenharia Elétrica e Computação, Universidade Federal do Pará, Pará, Brasil, E-mails: gilvan82@yahoo.com.br, jweyl@ufpa.br. Este trabalho foi financiado pelo CNPQ (06/56413-1).
Em virtude da aplicabilidade desses filtros existe um grande interesse, tanto do ponto de vista acadêmico, quanto industrial, no desenvolvimento de aplicativos para simulação (análise projeto) dessas estruturas. Existem várias técnicas para projeto de filtros ópticos, algumas delas clássicas, como projetos de filtros rejeita-faixa simples ou passa-faixa múltiplos com arranjo de estruturas de $\frac{1}{4}$ de comprimento de onda e cavidades Fabry-Perot, etc [3]. Entretanto, para projeto de filtros com perfis de refletividade arbitrários, o problema é mais complexo, altamente não linear e, desse modo, ainda há espaço para investigação de novas técnicas que auxiliem na solução e melhoria no projeto dessas estruturas [1]. Dada a característica não linear dessa classe de problema, a aplicação de técnicas baseadas em métodos probabilísticos de otimização vem sendo uma alternativa buscada [1].

Desde a década de 90, o PSO (Particle swarm optimization) foi proposto como uma ferramenta alternativa e de fácil implementação para resolver problemas de otimização. Onde tem mostrado ser eficiente em otimização multidimensional de problemas que apresentam descontinuidades, não-linearidades e são multimodais (possuem vários ótimos locais) [4]-[5]. Além disso, o mesmo foi aplicado de forma bem sucedida em problemas evolvendo eletromagnetismo [5][6]. Neste trabalho é proposta a aplicação de PSO para projeto de filtros ópticos multicamadas. Assim, são obtidos projetos de coberturas antirefletoras e filtros ópticos para sistemas de interrogação em sensoriamento óptico.

\section{DEFINIÇÃO DO PROBLEMA}

Os filtros multicamadas podem ser entendidos como um conjunto de $n$ camadas ou filmes de materiais diferentes superpostos, conforme mostra a Figura 1. De forma que projetar essas estruturas, consiste basicamente em procurar uma combinação adequada de valores (correspondente a parâmetros como índice de refração e espessura de cada uma das camadas) que representem um filtro cujo espectro de refletividade seja o mais próximo possível do desejado.

Com base nisso, cada combinação desses valores, corresponde a uma alternativa de solução no espaço de busca do problema (solução candidata), dado pela equação 1 , que pode ser boa ou não. Onde esta avaliação ou qualificação é medida pelo valor de uma função de mérito, cujo único objetivo é determinar o quão próximo se está da solução desejada.

Neste artigo, esta função é definida como o inverso do erro médio quadrático entre a curva de refletividade obtida pelo filtro (solução que está sendo analisada), e a curva de refletividade desejada para o mesmo. Como mostrado na equação 2 . 


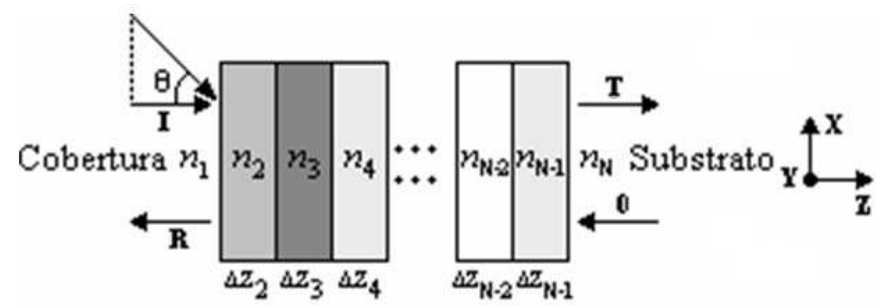

Fig. 1. Estrutura multicamadas.

$$
\begin{gathered}
x=\left(n_{2}, \Delta z_{2}, n_{3}, \Delta z_{3}, \cdots, n_{N-1}, \Delta z_{N-1}\right) \\
F(x) \hat{=} \frac{N_{A}}{\sum_{i=1}^{N_{A}}\left[\Gamma\left(\lambda_{i}, x\right)-\Gamma_{D}\left(\lambda_{i}\right)\right]^{2}}
\end{gathered}
$$

onde:

$x$ é o vetor de parâmetros, ou variáveis, do filtro. Representa um ponto no domínio da função $F(x)$;

$N_{A}$ é o número de amostras usadas para definir a curva de refletividade em função do comprimento de onda;

$i$ é o índice da amostra;

$\Gamma\left(\lambda_{i}, x\right)$ é o valor de refletividade, obtida pelo filtro $x$, no comprimento de onda $\lambda_{i}$;

$\Gamma_{D}\left(\lambda_{i}\right)$ é o valor de refletividade, desejada para o filtro a ser projetado, no comprimento de onda $\lambda_{i}$;

Ressalta-se que o método de análise de filtros multicamadas, usado neste artigo, para obtenção da curva de refletividade $\Gamma(\lambda, x)$ de um filtro $x$, necessária em 2 , foi o matricial, freqüentemente referenciado como método das matrizes características [7]-[8].

Cada ponto (definido na equação 1) no domínio da função $F(x)$, corresponde a uma possibilidade de filtro multicamadas, cuja imagem (definida na equação 2) o qualifica em relação ao filtro desejado. Logo, por uma visão matemática, o ato de projetar filtros se reduz simplesmente na otimização da função $F(x)$. Ou seja, o ponto no domínio cuja função $F(x)$ é máxima, corresponde ao filtro que mais se aproxima do almejado no projeto.

O método utilizado para maximizar a função $F(x)$, neste artigo, foi PSO, por ser uma ferramenta de otimização de bom desempenho com baixos requisitos computacionais, que veio a mostrar-se competitiva em relação a outras heurísticas já usadas em classes de problemas como esse, onde a função a ser otimizada é n-dimencional, não linear, e com muitos máximos e mínimos locais.

\section{PSO}

Este método de otimização estocástico foi desenvolvido na década de 90 por Kenedy e Eberthart através de uma metáfora do comportamento social de aves [9]. O mesmo é baseado em um modelo simplificado da teoria de enxames (swarm theory) onde bando de pássaros e cardumes de peixes, em busca de alimento em uma determinada região (região ótima), faz uso da experiência individual acumulada por cada individuo (partícula) bem como pelo resultado da experiência acumulada pelo grupo (enxame). Nesta metáfora, cada partícula corresponde a uma posição, ou alternativa de solução, no espaço de busca de um dado problema de otimização, onde as mesmas têm associado um valor indicando sua avaliação em relação à solução do problema, e um deslocamento que define a direção de seu movimento.

Estas partículas movem-se sob ação de três influências (vetores) que se compõem aditivamente, e que recebem os nomes de inércia, memória e cooperação. O primeiro vetor impele a partícula numa direção idêntica à que ela vinha seguindo. O segundo vetor atrai a partícula na direção da melhor posição até ao momento ocupado pela partícula durante a sua vida. $O$ terceiro vetor atrai a partícula na direção do melhor ponto do espaço até o momento descoberto pelo enxame.

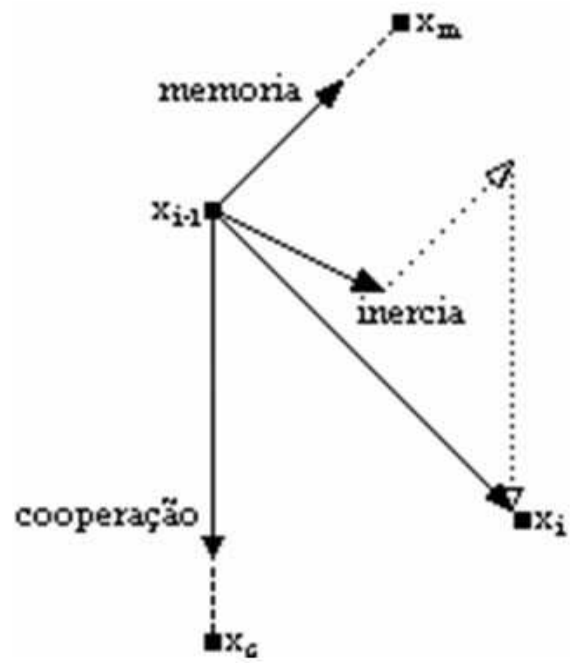

Fig. 2. Representação do movimento de uma partícula influenciada pelos vetores de inércia, memória e cooperação.

Esta técnica veio a mostrar-se competitiva em relação a outras metaheurísticas, haja vista que as aplicações presentes na literatura evidenciam a capacidade do algoritmo na solução de diferentes problemas, bem como salientam a habilidade de trabalhar com variáveis discretas e contínuas simultaneamente [5]. Tudo isso através de conceitos simples que envolvem baixos requisitos computacionais, já que todo o método baseiase em apenas duas fórmulas (considerando o algoritmo mais básico):

$d x_{i}=w \cdot d x_{i-1}+k_{1} \cdot r n d_{1}()\left(x_{m}-x_{i-1}\right)+k_{2} \cdot r n d_{2}() \cdot\left(x_{c}-x_{i-1}\right)$

$$
x_{i}=x_{i-1}+d x_{i}
$$

onde:

$i$ é o índice da iteração, com analogia ao intervalo de tempo atual;

$x_{i}$ é o vetor posição atual da partícula;

$x_{i-1}$ é o vetor posição anterior da partícula; 
$d x_{i}$ é o vetor deslocamento atual da partícula;

$d x_{i-1}$ é o vetor deslocamento anterior da partícula;

$x_{m}$ é a melhor posição encontrada pela partícula durante a sua vida;

$x_{c}$ é a melhor posição encontrada pelo enxame até aquele momento;

$r n d_{1}()$ e $r n d_{2}()$ são números aleatórios oriundos de uma distribuição uniforme no intervalo $[0,1]$. Os mesmos provocam uma perturbação na trajetória de cada partícula que se demonstrou ser benéfica para a exploração do espaço e a descoberta da solução ótima;

$w, k_{1}$ e $k_{2}$ são vetores pesos, em que cada elemento dos mesmos corresponde a um peso agindo em cada dimensão dos termos inércia, memória e cooperação respectivamente.

Inicialmente a cada partícula é atribuído um deslocamento e uma posição aleatórios, e a cada intervalo de tempo o deslocamento e a posição são atualizados de acordo com as equações 3 e 4 . O algoritmo pode ser simplificado nas etapas mostradas na figura 3.

\begin{tabular}{|l|}
\hline \multicolumn{1}{|c|}{ Algoritmo: } \\
Inicio \\
Inicialize o enxame (posição e deslocamento); \\
Repita \\
Atualize deslocamentos; \\
Atualize posições; \\
Avalie as novas posições; \\
Até critério de parada satisfeito; \\
Fim \\
\hline
\end{tabular}

Fig. 3. Algoritmo simplificado do PSO.

Para evitar que os deslocamentos de cada partícula cresçam de forma excessiva impossibilitando a convergência do enxame, costuma-se definir um limite para os mesmos $d x_{\max }$. Kennedy e Eberhart sugeriram em seu artigo que este valor seja o intervalo máximo de cada variável ou dimensão [10].

As experiências com o PSO simples rapidamente mostraram que, se o método parecia ter propriedades interessantes em convergir para a zona do ótimo, apresentava imensas dificuldades para afinar essa convergência, fundamentalmente porque a velocidade das partículas (determinada pelo seu deslocamento) continuava excessiva quando se pretendia que ela se suavizasse de forma a se aproximar cada vez com maior precisão do ponto ótimo.

Rapidamente se compreendeu que seria útil conceber um mecanismo que fosse gradualmente reduzindo a velocidade das partículas, à medida que essas se aproximassem da solução final. Um dos mecanismos mais utilizados para esse fim, já que o principal agente deste mau comportamento é o termo de inércia, corresponde em aplicar uma função decrescente no tempo afetando este termo. Erberhard e Shi propuseram, de forma empírica, uma variação linear do coeficiente de inércia de 0.9 a 0.4 [11].

\section{RESUltado DAS SIMULAÇÕES E DiscuRSÃO}

Nesta seção, encontra-se 4 projetos de filtros ópticos multicamadas utilizando o PSO como método de otimização. Em todos eles, foram empregados na composição das camadas, apenas um entre dois tipos de materiais de índices de refração diferentes. Para efeito de comparação dos resultados, a escolha desses materiais, assim como as especificações de cada um dos projetos, foram feitas com base em outros projetos semelhantes encontrados na literatura [1][12].

Nos dois primeiros projetos, cada camada deverá ser constituída por qualquer um dos dois tipos de materiais predefinidos, sem nem uma restrição. Portanto existe a possibilidade de uma camada possuir o mesmo tipo de material de sua adjacente, formando assim, do ponto de vista físico, uma única camada. Isto seria uma forma implícita de otimizar o número de camadas do filtro, apresentando bons resultados quando este número, definido inicialmente, for pequeno.

Nos dois últimos projetos, cada camada deverá ser constituída por um dos dois tipos de materiais predefinidos, dispostos obrigatoriamente de forma alternada, de modo que o filtro projetado terá o mesmo número de camadas definido inicialmente. Logo, não existirá uma otimização do número de camadas, porém haverá uma redução do número de variáveis a serem otimizadas.

\section{A. Coberturas antirefletoras}

$\mathrm{O}$ projeto 1 e o projeto 2 , consistem de filtros ópticos chamados de coberturas antirefletoras. Essas coberturas são normalmente projetadas para atuarem em regiões espectrais que se estendem desde o ultravioleta até o infravermelho.

A razão tecnológica para implementação de coberturas antirefletoras que operem nas faixas de freqüência definidas nesses projetos, consiste no fato de que, os materiais ópticos utilizados nessa parte do espectro eletromagnético, são semicondutores com altos valores de índices de refração, o que provoca altos valores de refletividade [12].

1) Projeto 1: O objetivo é reduzir a zero a refletividade de um substrato de índice de refração 4 , na região espectral que vai de $7.7 \mu \mathrm{m}$ a $12.3 \mu \mathrm{m}$. As especificações deste projeto são detalhadas na tabela I.

TABELA I

PARÂMETROS DO PROJETO 1.

\begin{tabular}{|c|c|}
\hline Faixa espectral & $7.7 \mu \mathrm{m} \mathrm{a} 12.3 \mu \mathrm{m}$ \\
\hline Ângulo de incidência & $0^{\circ}$ \\
\hline Cobertura & 1.0 \\
\hline Substrato & 4.0 \\
\hline Índices de refração & $2.2 \mathrm{e} 4.2$ \\
\hline Número de camadas & 4 \\
\hline Espessura mínima por camada & $0.01 \mu \mathrm{m}$ \\
\hline Espessura máxima por camada & $2.8 \mu \mathrm{m}$ \\
\hline
\end{tabular}

A figura 4 mostra a curva de refletividade otimizada, representando uma cobertura antirefletora cuja refletividade média é $2.1701 \%$.

A figura 5 exibe o perfil de índice de refração do filtro sintetizado. Onde o número de camadas era originalmente igual a 4, fixado antes da aplicação do processo de otimização, 


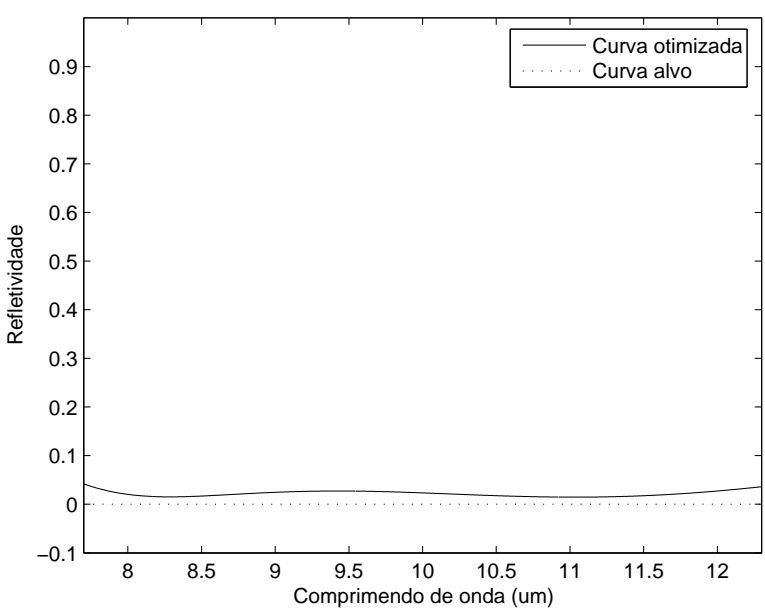

Fig. 4. Espectro de refletividade do filtro, obtido no projeto 1.

conforme mostrado na tabela I. Mas durante o processo de otimização, camadas contíguas apresentaram o mesmo valor de índice de refração, constituindo assim uma única camada, e por conseqüência diminuindo o número de camadas efetivas do dispositivo.

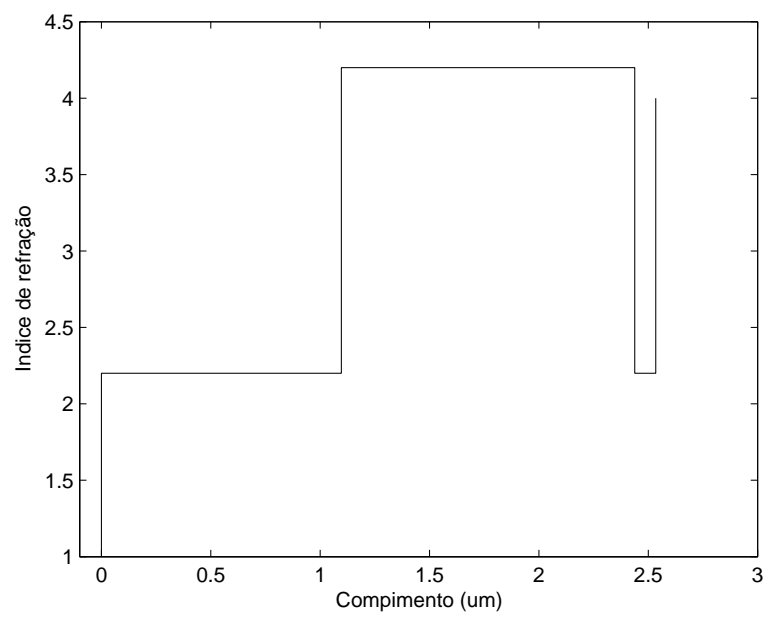

Fig. 5. Perfil de índice de refração do filtro, obtido no projeto 1.

2) Projeto 2: Este projeto também consiste na síntese de uma cobertura antirefletora de um substrato de índice de refração 4, porém em outra região do espectro eletromagnético $(2.5 \mu \mathrm{m}$ a $5.5 \mu \mathrm{m})$, usando outros materiais, etc. As especificações deste novo projeto encontram-se na tabela II.

A figura 6 mostra o espectro de refletividade do filtro otimizado, tendo como resultado uma cobertura antirefletora cuja refletividade média é de apenas $0.9255 \%$, e a figura 7 mostra o perfil de índice de refração encontrado.

O projeto apresenta como resposta um filtro bem simples, com apenas 2 camadas. Como no projeto 1, camadas contíguas apresentaram o mesmo valor de índice de refração, constituindo assim apenas uma camada. O número de camadas
TABELA II

PARÂMETROS DO PROJETO 2.

\begin{tabular}{|c|c|}
\hline Faixa espectral & $2.8 \mu \mathrm{m} \mathrm{a} 5.5 \mu \mathrm{m}$ \\
\hline Ângulo de incidência & $0^{\circ}$ \\
\hline Cobertura & 1.0 \\
\hline Substrato & 4.0 \\
\hline Índices de refração & $1.35 \mathrm{e} 2.4$ \\
\hline Número de camadas & 8 \\
\hline Espessura mínima por camada & $0.01 \mu \mathrm{m}$ \\
\hline Espessura máxima por camada & $1.0 \mu \mathrm{m}$ \\
\hline
\end{tabular}

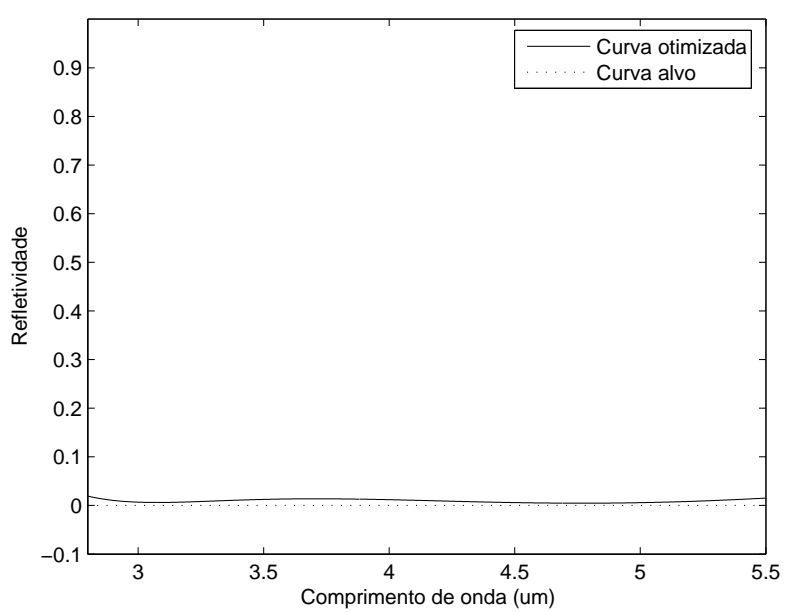

Fig. 6. Espectro de refletividade do filtro, obtido no projeto 2

originalmente configurado era igual a 8 , conforme mostrado na tabela II.

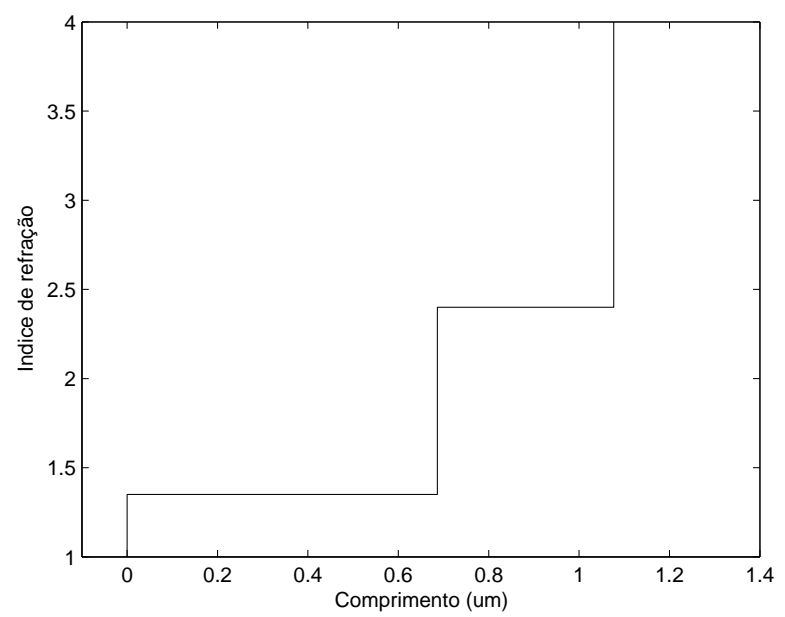

Fig. 7. Perfil de índice de refração do filtro, obtido no projeto 2

\section{B. Filtro óptico para aplicações a sensores}

Os filtros ópticos multicamadas podem ser aplicados na interrogação de comprimento de onda em sistema de sensoriamento. Através de espectros em forma de rampa, pode-se converter grandezas expressas por desvios na freqüência para 
grandezas expressas por variações de potência ótica, que é algo mais simples de detectar [1].

1) projeto 3: Este projeto trata de um filtro imerso no ar cujo espectro de refletividade se assemelha a uma rampa ascendente no intervalo de $0.4 \mu \mathrm{m}$ a $0.7 \mu \mathrm{m}$, possuindo refletividades mínima e máxima de $0 \%$ e $100 \%$ respectivamente. As especificações do projeto encontram-se na tabela III, a curva de refletividade obtida, na figura 8 , e o perfil de índice de refração na figura 9.

TABELA III

PARÂMETROS DO PROJETO 3.

\begin{tabular}{|c|c|}
\hline Faixa espectral & $0.4 \mu \mathrm{m} \mathrm{a} 0.7 \mu \mathrm{m}$ \\
\hline Ângulo de incidência & $0^{\circ}$ \\
\hline Cobertura & 1.0 \\
\hline Substrato & 1.0 \\
\hline Índices de refração & $1.0583 \mathrm{e} 1.68$ \\
\hline Número de camadas & 15 \\
\hline Espessura mínima por camada & $0.05 \mu \mathrm{m}$ \\
\hline Espessura máxima por camada & $0.33 \mu \mathrm{m}$ \\
\hline
\end{tabular}

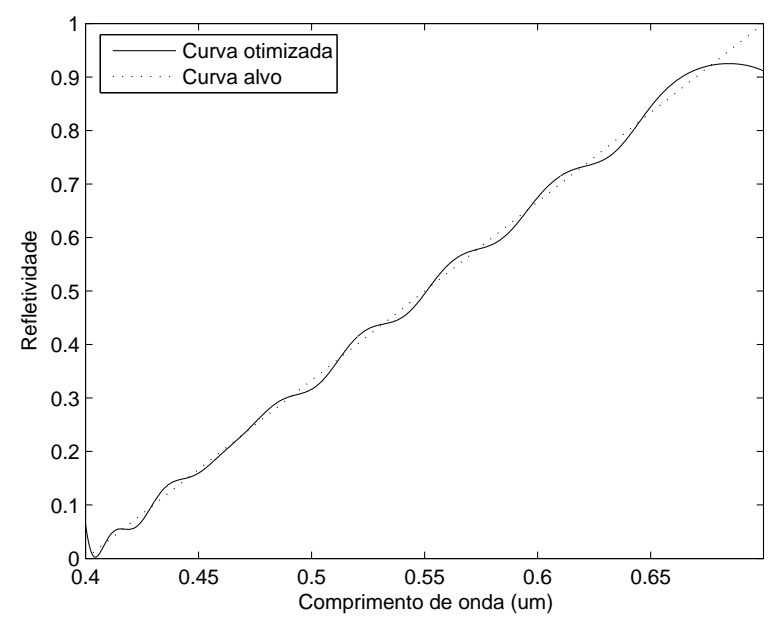

Fig. 8. Espectro de refletividade do filtro, obtido no projeto 3

\section{Filtros ópticos não convencionais}

Nessa seção é apresentado um projeto que apesar de não mostrar aplicações práticas, consiste em um desafio para o algoritmo de otimização. E assim pode-se avaliar a eficiência da técnica para projetos não convencionais.

1) Projeto 4: Neste projeto deseja-se um filtro imerso no ar capaz de refletir $50 \%$ da energia luminosa de faixa azul (considerando o seu comprimento de onda variando de 0.4 $\mu \mathrm{m}$ a $0.5 \mu \mathrm{m}$ ) e $100 \%$ da energia de luz vermelha (com o seu comprimento de onda considerado entre $0.6 \mu \mathrm{m}$ e $0.7 \mu \mathrm{m}$ ), entre essas faixas, de $0.5 \mu \mathrm{m}$ a $0.6 \mu \mathrm{m}$, o mesmo deverá ser transparente. Abaixo temos as especificações do projeto (tabela IV), a correspondente curva de refletividade encontrada (figura 10), e o perfil de índice de refração do filtro sintetizado (figura 11).

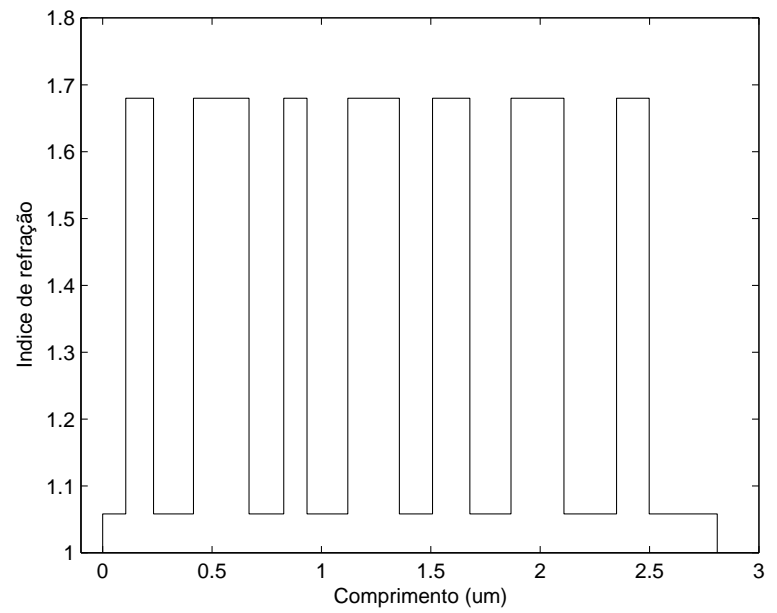

Fig. 9. Perfil de índice de refração do filtro, obtido no projeto 3

TABELA IV

PARÂMETROS DO PROJETO 4.

\begin{tabular}{|c|c|}
\hline Faixa espectral & $0.4 \mu \mathrm{m} \mathrm{a} 0.7 \mu \mathrm{m}$ \\
\hline Ângulo de incidência & $0^{\circ}$ \\
\hline Cobertura & 1.0 \\
\hline Substrato & 1.0 \\
\hline Índices de refração & $1.46 \mathrm{e} 2.1$ \\
\hline Número de camadas & 20 \\
\hline Espessura mínima por camada & $0.045 \mu \mathrm{m}$ \\
\hline Espessura máxima por camada & $0.24 \mu \mathrm{m}$ \\
\hline
\end{tabular}

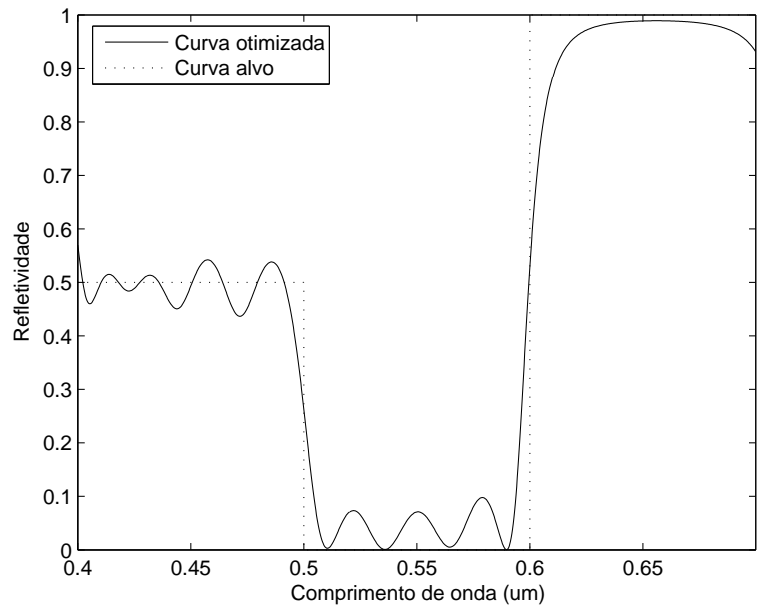

Fig. 10. Espectro de refletividade do filtro, obtido no projeto 4

\section{Conclusões}

Foi apresentado um método baseado em particle swarm optimization para o projeto de filtros ópticos multicamadas. Como se pôde observar, o PSO é promissor como algoritmo de otimização para síntese de filtros ópticos, com a vantagem de ser relativamente simples e necessitar de baixos requisitos computacionais, comparados a outras heurísticas, além de possuir poucos parâmetros de ajuste. Além disso, o algoritmo 


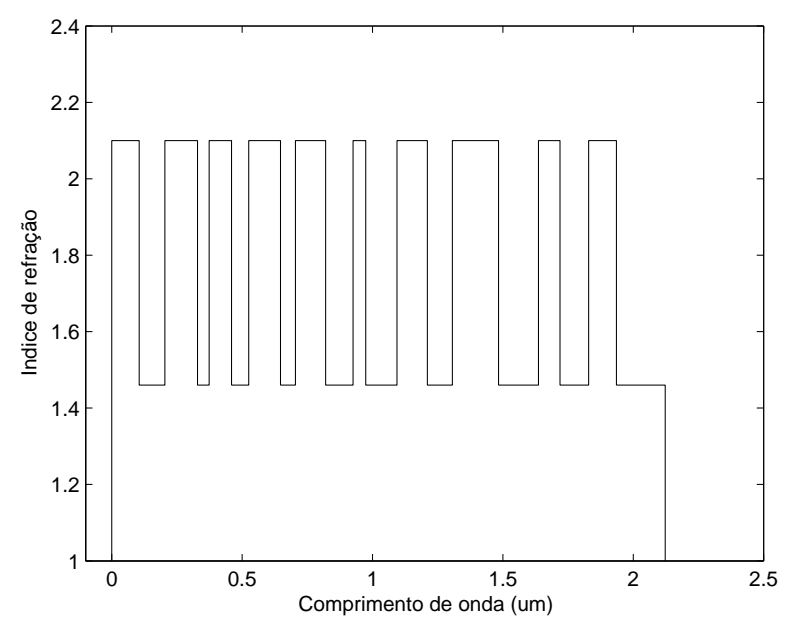

Fig. 11. Perfil de índice de refração do filtro, obtido no projeto 4

pôde se adequar a requisitos de projetos bastante diversificados, desde projetos simples de coberturas antirefletoras até filtros de espectros mais complexos na forma de rampa. Os perfis de índice de refração obtidos apresentaram pouca complexidade, facilitando assim a fabricação desses dispositivos na prática.

\section{AGRADECIMENTOS}

O Laboratório de Eletromagnetismo Aplicado agradece ao CNPQ pelo apoio financeiro durante a execução deste trabalho.

\section{REFERÊNCIAS}

[1] M. J. Sousa, Síntese de grades de Bragg utilizando algoritmo genético e processamento paralelo, Universidade Federal do Pará - UFPA, 2003.

[2] A. Chandran, Self-Assembled multilayered dielectric spectral filters, Faculty of Virginia Polytechnic Institute, 2001.

[3] S. A. Furman e A.V. Tikhonravov, Book Review: Basics of optics and multilayer systems, Edition Frontieres, 1992.

[4] J. A. C. C. Medeiros, Enxame de partículas como ferramenta de otimização em problemas complexos de engenharia nuclear, Edition Frontieres, 2005.

[5] M. G. dos Santos, L. S. Ochi, M. Th. Schilling, M. B. do Coutto e J. C. S. de Souza, "Controle ótimo de tensão em sistemas de potência utilizando nuvem de partículas" Sba Controle \& Automação, vol. 17, n. 3, Campinas jul./set. 2006.

[6] J. Robinson e Y. Rahmat-Samii, "Particle Swarm Optimization in electromagnetics," IEEE Transactions on Antennas and Propagation, vol.52, pp. 397-407, 2004.

[7] S. T. Peng, Theodor Tamir, e Henry L. Bertoni, "Theory of periodic dielectric waveguides," IEEE Transactions on Microwave Theory and Techniques, vol. 23, pp. 123-133, 1975.

[8] M. Born and E. Wolf, Principles of Optics, New York: Pergamon, 1987.

[9] J. Kennedy e R. Eberhart, "Particle Swarm Optimization," Proceedings of the 1995 IEEE International Conference on Neural Networks, vol. 4, pp. 1942-1948, 1995.

[10] J. Kennedy e R. Eberhart, "A new optimizer using Particle Swarm Theory," Sixth International Symposium on Micro Machine and Human science, pp. 39-43, 1995.

[11] R. C. Eberhart e Y. Shi, "Comparing inertia weights and constriction factors in Particle Swarm Optimization," Proceedings of the 2000 Congress on Evolutionary Computation, pp. 84-88, Washington, DC, 2000.

[12] J. C. C. Carvalho, Algoritmos genéticos aplicados a síntese de dispositivos ópticos, Universidade Federal do Pará - UFPA, 1999.

[13] H. T. Hattori, Sistemas interrogadores de sensores baseados em grade de Bragg, Instituto Tecnológico da Aeronáutica - ITA, 1999. 